\title{
Intervenciones educativas para la inclusión en el aula de estudiantes con aptitudes sobresalientes
}

\section{Educational interventions for the inclusion in the classroom of students with high abilities}

\author{
GUTIÉRREZ-LADINO, Leidy†*1, LÓPEZ-AYMES, Gabriela ${ }^{2}$, MORENO-AGUIRRE, Alma ${ }^{1}$ y \\ ORTÍZ-RODRÍGUEZ, María Araceli*3
}

\author{
${ }^{\text {I}}$ Facultad de Comunicación Humana, Universidad Autónoma del Estado de Morelos (UAEM), México. \\ ${ }^{2}$ Centro de Investigación Transdisciplinar en Psicología (CITPsi), Universidad Autónoma del Estado de Morelos (UAEM), \\ México
}

${ }^{3}$ Facultad de Nutrición, Universidad Autónoma del Estado de Morelos (UAEM), México

ID $1^{\mathrm{er}}$ Autor: Leidy, Gutiérrez-Ladino / ORC ID: 0000-0001-9404-5557 CVU CONACYT ID: 1027553

ID $1^{\mathrm{er}}$ Coautor: Gabriela, López-Aymes / ORC ID: 0000-0002-4634-1973, CVU CONACYT ID: 78924

ID $2^{\text {do }}$ Coautor: Alma, Moreno-Aguirre / ORC ID: 0000-0001-9076-5987, CVU CONACYT ID: 173543

ID $3^{\text {er }}$ Coautor: María Araceli, Ortiz-Rodríguez / ORC ID: 0000-0003-0847-0261, Researcher ID Thomson: T-3707-2018, CVU CONACYT ID: 449164

DOI: $10.35429 / J E S C .2020 .12 .4 .6 .11$

Recibido: 04 de Octubre, 2020; Aceptado 22 de Noviembre, 2020

\section{Resumen}

La atención educativa de estudiantes con aptitudes sobresalientes, en la escuela regular, se ha convertido en un gran reto para el sistema educativo mexicano, por tanto, el objetivo de este artículo es revisar en diferentes fuentes documentales, investigaciones llevadas a cabo en México durante el periodo de 2010-2020 donde se ponga en evidencia los programas o estrategias de intervención dirigidos a esta población. Para tal fin, el diseño de esta investigación es de tipo descriptivo con una búsqueda de literatura científica que ha comprendido el periodo de tiempo 2010-2020 en las bases de datos Researchgate, Scielo, Scopus, Dialnet y Redalyc. Adicionalmente, se ha considerado la información oficial de la Secretaría de Educación Pública de México (SEP) y una revisión bibliográfica en bibliotecas nacionales. Una vez alcanzado el objetivo anterior se espera que el conocimiento docente de las estrategias de intervención educativa y los programas de intervención aquí expuestos favorezcan el diseño y la implementación de ajustes curriculares para la inclusión educativa de estudiantes con aptitudes sobresalientes en escuelas regulares.

Aptitudes sobresalientes, Intervención educativa, Educación inclusiva

\begin{abstract}
The educational attention of students with outstanding abilities, in the regular school, has become a great challenge for the Mexican educational system, therefore, the objective of this article is to review in different documentary sources, investigations carried out in Mexico during the period 20102020 where the intervention programs or strategies aimed at this population are highlighted. To this end, the design of this research is descriptive with a search of scientific literature that has included the period of time 2010-2020 in the Researchgate, Scielo, Scopus, Dialnet and Redalyc databases. In addition, the official information of the Secretariat of Public Education of Mexico (SEP) and a bibliographic review in national libraries have been considered. Once the previous objective has been achieved, it is expected that the teacher's knowledge of the educational intervention strategies and the intervention programs presented here will favor the design and implementation of curricular adjustments for the educational inclusion of students with high abilities in regular schools.
\end{abstract}

High abilities, Educational intervention, Inclusive education

Citación: GUTIÉRREZ-LADINO, Leidy, LÓPEZ-AYMES, Gabriela, MORENO-AGUIRRE, Alma y ORTÍZRODRÍGUEZ, María Araceli. Intervenciones educativas para la inclusión en el aula de estudiantes con aptitudes sobresalientes. Revista Ciencias de la Educación. 2020. 4-12: 6-12

\footnotetext{
* Correspondencia del Autor (correo electrónico: araceli.ortiz@uaem.mx)

$\dagger$ Investigador contribuyendo como primer autor.
} 


\section{Introducción}

La Secretaría de Educación Pública de México (SEP) define al alumnado con aptitudes sobresalientes como "aquéllos/as capaces de destacar significativamente del grupo social y educativo al que pertenecen, en uno o más de los siguientes campos del quehacer humano: científico-tecnológico, humanístico-social, artístico o de acción motriz, pero al presentar necesidades específicas requieren de un contexto facilitador que les permita desarrollar sus capacidades personales y satisfacer sus necesidades e intereses para su propio beneficio y el de la sociedad" (SEP, 2011).

Dentro de estos campos, la nominación dada por la SEP a dichas aptitudes sobresalientes, es: aptitud sobresaliente intelectual; aptitud sobresaliente creativa; aptitud sobresaliente psicomotriz; aptitud sobresaliente artística y aptitud sobresaliente socio afectiva (SEP, 2006).

A nivel teórico la atención educativa a dichos estudiantes se puede abordar a partir de la pedagogía diferencial la cual concibe al estudiantado como sujeto de la educación (Tourón, 2020) y atiende la diversidad en el aula (Jiménez y González, 2011).

Dentro de esta pedagogía se puede ubicar, entre otras estrategias de intervención, las siguientes: a) el agrupamiento (Pérez y Jiménez, 2018; Walker et al., 2011); b) el enriquecimiento (SEP, 2006) y b) la aceleración (Sánchez, 2010; Villegas et al., 2016).

En México, dichas estrategias se recogen en la Propuesta de intervención: Atención educativa a alumnos y alumnas con aptitudes sobresalientes de la SEP (2006), y es a partir del año 2008 que se comienzan a implementar en todo el territorio nacional. A pesar de que existe un respaldo académico y legislativo para la atención de esta población, se conoce aún poco el tipo de estrategias de atención educativa que con más frecuencia se han implementado en los contextos escolares y extraescolares, así como su evaluación. Por lo tanto, el objetivo de este estudio es revisar en diferentes fuentes documentales, investigaciones llevadas a cabo en México durante el periodo de 2010-2020 donde se ponga en evidencia los programas o estrategias de intervención dirigidos a esta población.

\section{Metodología}

El diseño de esta investigación es de tipo descriptivo con una búsqueda de literatura científica que ha comprendido el periodo de tiempo 2010-2020.

\section{Descriptores usados}

La revisión de literatura científica se ha llevado a cabo bajo los siguientes descriptores: aptitudes sobresalientes, altas capacidades, superdotación, alta habilidad, alto potencial, estudiantes excepcionales, alumnos superdotados, aptitud intelectual superior, sobrecapacidad, alta habilidad, sobredotación, intervención educativa, enriquecimiento, aceleración, agrupamiento, trabajo cooperativo, creatividad y nombres de autores mexicanos.

En el transcurso de la búsqueda los descriptores se fueron combinando para garantizar la coherencia y la cohesión con el tema investigado.

\section{Bases de datos usadas}

Researchgate, Scielo, Scopus, Dialnet y Redalyc.

Adicionalmente, se ha considerado la búsqueda en la página de la Secretaría de Educación Pública de México (SEP) y en bibliotecas nacionales.

\section{Criterios para la inclusión de publicaciones}

- $\quad$ País de origen de la población de estudio: México.

- $\quad$ Año de publicación: 2010 al 2020.

- Tema de publicación: intervención y evaluación de intervenciones para la atención de estudiantes con aptitudes sobresalientes.

- $\quad$ Presencia de autores mexicanos.

\section{Procedimiento}

1. Búsqueda en las bases de datos mencionadas arriba.

2. Revisión de libros o capítulos sobre el tema de publicación.

3. Búsqueda de publicaciones, en las bases de datos, por nombre de autores mexicanos reportados en la búsqueda inicial. 
4. Eliminación de duplicados.

Y por último, los resultados fueron filtrados teniendo en cuenta el cumplimiento de los criterios de inclusión.

\section{Resultados}

Esta revisión de literatura científica arrojó un total de 112 resultados de los cuales el $30.35 \%$ cumplió con los criterios de inclusión nombrados anteriormente.

Este porcentaje fue categorizado teniendo en cuenta las diferentes estrategias de intervención que se pueden usar para atender estudiantes con aptitudes sobresalientes. Dichas categorías son: a) agrupamiento; b) enriquecimiento áulico; c) enriquecimiento escolar; d) enriquecimiento extraescolar; e) aceleración; y adicionalmente se creó la categoría f) enriquecimiento combinado para incluir ahí aquellos programas donde se usó más de un tipo de estrategia de intervención.

\section{Estas categorías obtuvieron los} siguientes resultados:

a) agrupamiento: $2.94 \%$ (Valadez et al., 2020); b) enriquecimiento áulico: $20.6 \%$ (Betancourt y Valadez, 2016; Molinero et al., 2016; Valadez et al., 2015); c) enriquecimiento escolar: 11,76\% (Ordaz, Acle y Antonio, 2016; Ordaz, Acle y Armenta, 2016; Ruíz et al., 2017; Zacatelco et al., 2017); d) enriquecimiento extraescolar: 41.2\% (Acle et al., 2015; Antonio et al., 2015; Antonio y Acle, 2013; Bello et al., 2013; Borges et al., 2016; Chávez y Zacatelco, 2013; De la Torre, 2016; López, 2015; LópezAymes et al., 2020; López-Aymes et al., 2016; López-Aymes y Acuña, 2012; Molinero et al., 2012; Tepetla et al., 2013; Zacatelco y Chávez, 2012); e) aceleración: 5.88\% (De Alba et al., 2016; Verdín et al., 2012); y f) enriquecimiento combinado: $17.65 \%$ (Covarrubias, 2014; Covarrubias y Marín, 2015; González, 2016; Tuyub et al., 2016; Valadez et al., 2016; Zambrano, 2017).

\section{Productividad temporal}

El año 2016 fue en el cual hubo una mayor cantidad de evidencia la cual quedó representada por el $41.2 \%$ del total.
Adicionalmente, la media de publicaciones por año fue de $3.4 \%$ la cual se alcanzó y en algunos casos se superó en los años: 2012, 2013, 2015, 2016, 2017 y 2020.

\section{Agradecimiento}

Este estudio fue financiado por una beca de Posgrados de Calidad (PNPC) del Consejo Nacional de Ciencia y Tecnología de México (CONACYT) otorgada por medio de la Universidad Autónoma del Estado de Morelos (UAEM).

\section{Conclusiones}

La revisión de literatura científica, en su etapa inicial, mostró un gran porcentaje de trabajos descriptivos, por tanto, se espera que la media de publicaciones por año vaya en aumento en lo que respecta a investigaciones cuasi experimentales.

Por otro lado, los datos evidencian la falta de programas de enriquecimiento áulico, lo cual llama la atención porque para que la inclusión educativa de personas con aptitudes sobresalientes se lleve a cabo, el salón de clases debe ser el protagonista por excelencia.

Y es en dicho lugar donde la pedagogía diferencial incentiva el uso de nuevas técnicas de enseñanza, de espacios poco frecuentados en la escuela, materiales diversos, trabajo en grupos, entre otros, tanto para atender a estudiantes con y sin aptitudes sobresalientes (SEP, 2006).

Al respecto, probablemente, las estrategias que se implementan en el aula no son reportadas en los estudios realizados por los investigadores.

Por otro lado, a pesar que se encontraron pocas evidencias sobre la estrategia de agrupamiento, se debe tener en cuenta que en la mayoría de programas de intervención esta estrategia de intervención está incluida de manera intrínseca en el desarrollo de las actividades, lo cual permite que los estudiantes, mejoren la motivación académica, las habilidades socioemocionales, el pensamiento crítico y el rendimiento académico, entre otros (Moruno et al., 2011; Valadez et al., 2020). 
Por su parte, a pesar que la aceleración, en México, se concretizó a nivel legal por medio del acuerdo 648 aún parece una materia pendiente en el sector educativo (De Alba et al., 2016).

En este contexto, para futuras investigaciones se recomienda hacer distinción entre el grado académico que cubren los programas de intervención para saber qué grados están pendientes de atender a nivel científico.

\section{Referencias}

Acle, G., Martínez, L., Lozada, R. y Ordaz, G. (2015). Social validity by parents of special education programs based on the ecological risk/resilience model. Acta Colombiana de Psicologia, 18(2),

151-161. https://www.researchgate.net/publication/28297 8255_Social_validity_by_parents_of_special_e ducation_programs_based_on_the_ecological_r iskresilience_model

Antonio, A., Acle, G. Martínez, L. y Ordaz, G. (2015). Social Validity of a Creative and Cognitive Enrichment Program for Gifted Children. Creative Education, 6, 1667-1679. https://www.researchgate.net/publication/28296 3779_Social_Validity_of_a_Creative_and_Cog nitive_Enrichment_Program_for_Gifted_Childr en

Antonio, A., y Acle, G. (2013). Programa de enriquecimiento cognitivo y creativo con niños sobresalientes desde un modelo ecológico riesgo/resiliencia. En J. Gázquez, M. d. C., Pérez, M. d. M, Molero y R. Parra (Comps.). Investigación en el ámbito escolar: un acercamiento multidimensional a las variables psicológicas y educativas (387-392). GEU Editorial.

Bello, J., Chávez, B. y Zacatelco, F. (2013). Efectos de un programa para favorecer habilidades cognoscitivas básicas en un grupo de alumnos sobresalientes. En J. Gázquez, M. Pérez, M. Molero y R. Parra (Comps.). Investigación en el ámbito escolar: un acercamiento multidimensional a las variables psicológicas y educativas (17-22). GEU Editorial.
Betancourt, J. y Valadez, M. (2016). Planeta crea: programa para el desarrollo del pensamiento creativo de estudiantes con aptitudes sobresalientes. En M. Valadez, G. López-Aymes, A. Borges, J. Betancourt y R. Zambrano (Coords.). Programas de intervención para niños con altas capacidades y su evaluación. Manual Moderno.

Borges, A., Nieto, I., Moreno, C. y LópezAymes, G. (2016). Medida de la efectividad del Programa Integral para Altas Capacidades (PIPAC): expectativas del programa para padres en las ediciones de España y México. Talincrea, 32-45.

https://www.researchgate.net/publication/30151 4782_Medida_de_la_efectividad_del_Programa _Integral_para_Altas_Capacidades_PIPAC_exp ectativas_del_programa_para_padres_en_las_e diciones_de_Espana_y_Mexico

Chávez, B. y Zacatelco, F. (2013). Enriquecimiento de la creatividad: alternativas para promover conductas resilientes en niños sobresalientes de segundo ciclo de primaria. En G. Acle (coord.). Resiliencia en educación especial: una experiencia en la escuela regular (395-432). Gedisa.

Covarrubias, P. (2014). Alumnos sobresalientes: visión en torno a la atención que reciben. $I E$ Revista de Investigación Educativa de la REDIECH, 5(8), 21-28. https://www.redalyc.org/articulo.oa?id=521651 962004

Covarrubias, P. y Marín, R. (2015). Evaluación de la Propuesta de Intervención para Estudiantes Sobresalientes: Caso Chihuahua, México. Actualidades Investigativas en Educación 15(3), $1-32$.

https://www.researchgate.net/publication/28245 0786_Evaluacion_de_la_Propuesta_de_Interve ncion_para_Estudiantes_Sobresalientes_Caso_ Chihuahua_Mexico

De Alba, E., Valadez, M., Borges, M., Solter, R. y Zambrano, R. (2016). Evaluación del proceso de Acreditación, Promoción y Certificación Anticipada para niños con Aptitudes Sobresalientes Intelectuales. Revista Sobredotação, $\quad$ l(15), $\quad$ 131-144. https://www.aneis.org/wpcontent/uploads/2017/01/sobredotacao_2016.pd $\mathrm{f}$ \#page $=66$ 
De la Torre, G., Del Valle, L., Mijangos, A. y Williams, M. (2016). Programa Adopte un Talento. Impulso al talento científico. En M. Valadez, G. López-Aymes, A. Borges, J. Betancourt y R. Zambrano (Coords.). Programas de intervención para niños con altas capacidades y su evaluación. Manual Moderno.

González, L. (2016) Programa universitario para la atención a estudiantes sobresalientes de la Universidad Autónoma de Chiapas: YAS N'A. En M. Valadez, G. López-Aymes, A. Borges, J. Betancourt, y R. Zambrano (Coords.). Programas de intervención para niños con altas capacidades y su evaluación. Manual Moderno.

Jiménez, C. y González, M. (Coord.). (2011). Pedagogía diferencial y atención a la diversidad. Editorial Universitaria Ramón Areces.

López, V. (2015). Programa apoyo a sobresalientes en el estado de Sinaloa: una atención oportuna al talento. Ra Ximhai, 11(3), 19-34.

https://www.redalyc.org/articulo.oa?id=461354 09002

López-Aymes, G. y Acuña, S. (2012). Desarrollo emocional de niños con altas capacidades a través de un taller de expresión corporal. Revista de Investigación y Divulgación en Psicología y Logopedia, 2(2), 47-54. https://dialnet.unirioja.es/servlet/articulo?codig $\mathrm{o}=6369132$

López-Aymes, G., Navarro, M. y Vázquez, N. (2016). Evaluación formativa de la implementación del Programa Integral para Altas Capacidades en México desde el punto de vista de las monitoras. Talincrea. Talento, Inteligencia y Creatividad, 2(2), 46-67. https://www.researchgate.net/publication/30151 4946_Evaluacion_formativa_de_la_implementa cion_del_Programa_Integral_para_Altas_Capac idades_en_Mexico_desde_el_punto_de_vista_d e_las_monitoras

López-Aymes, G., Acuña, S. y Villegas, G. (2020). Resilience and creativity in teenagers with high intellectual abilities. A middle school enrichment experience in vulnerable contexts. Sustainability, 12(18), 7670.

https://www.scopus.com/authid/detail.uri?autho $\mathrm{rId}=57205607030$
Molinero, B., López-Aymes, G. y Acuña, S. (2012). Programa para desarrollar habilidades sociales a través del juego y la creatividad. En M. Gómez (presidencia), $1^{\text {er }}$ Congreso Internacional de Intervención Educativa. Guadalajara, Jal. 433-456. http://upnmorelos.edu.mx/2013/documentos_de scarga_2013/libro_electronico_lie.pdf

Molinero, B., López-Aymes, G. y Acuña, S. (2016). Programa Imagino, Creo y Aprendo. En M. Valadez, G. López-Aymes, A. Borges, J. Betancourt, y R. Zambrano (Coords.). Programas de intervención para niños con altas capacidades y su evaluación (Cap. 1). Manual moderno.

Moruno, P., Sánchez, M. y Zariquiey, F. (2011). La cultura de la cooperación. El aprendizaje cooperativo como herramienta de diferenciación curricular. En J. Torrego (coord.). (Cap. 6). Alumnos con altas capacidades y aprendizaje cooperativo. Un modelo de respuesta educativa, 167-197. Universidad de Alcalá. https://www.orientacionandujar.es/wpcontent/uploads/2014/07/Altas-capacidades-yaprendizaje-cooperativo.pdf

Ordaz, G., Acle, G. y Armenta, M. (2016). Crear con Ciencia: programa de enriquecimiento para el desarrollo de la creatividad mediante experimentos científicos. En M. Valadez., G. López-Aymes, A. Borges, J. Betancourt, y R. Zambrano (Coords.). Programas de intervención para niños con altas capacidades y su evaluación. Manual Moderno.

Ordaz, G., Acle, G. y Antonio, A. (2016). Experimentos científicos: actividades para promover la creatividad en alumnos con aptitudes sobresalientes. En Asociación Científica de Psicología y Educación. Psicología y Educación. Presente y Futuro (1359-1366). Juan Luis Castejón-Costa. https://www.researchgate.net/publication/31173 3759_Experimentos_cientificos_actividades_pa ra_promover_la_creatividad_en_alumnos_con_ aptitudes_sobresalientes

Pérez, L y Jiménez, C. (2018). Influencia de la organización escolar en la educación de los alumnos de altas capacidades. Enseñanza \& Teaching, 36(1), 151-178. https://doi.org/10.14201/et2018361151178 
Ruíz, L., González, A., Chávez, S. y Zacatelco, F. (2017). Intervención educativa para alumnos con aptitudes sobresalientes: desarrollo de la habilidad escrita. En L. Dubini, M. Erice, D. Meziat, M. García, L. Bengochea (eds.) Educación científica e inclusión sociodigital: actas del IX Congreso Iberoamericano de Educación Científica y del I Seminario de Inclusión Educativa y Sociodigital (CIEDUC 2017) Vol. 3 (1397-1405). https://dialnet.unirioja.es/servlet/articulo?codigo= 6320964

Sánchez, P. (2010). Percepciones de la aceleración de niños sobresalientes en escuelas mexicanas. En R. López (Presidencia), $X$ congreso nacional de investigación educativa: área 1: aprendizaje y desarrollo humanos (1-12). http://www.comie.org.mx/congreso/memoriaele ctronica/v10/pdf/area_tematica_01/ponencias/0 316-F.pdf

Secretaría de Educación Pública de México. (2006). Propuesta de intervención: Atención educativa alumnos y alumnas con aptitudes sobresalientes.

Autor. https://www.educacionespecial.sep.gob.mx/pdf/ aptitudes/intervencion/Propuesta_inter.pdf

Secretaría de Educación Pública de México. (2011). Atención educativa a alumnos con aptitudes sobresalientes. Guía para las familias. Autor.

https://www.educacionespecial.sep.gob.mx/pdf/ tabinicio/2012/guia_para_familias.pdf

Tepetla, F., Chávez, B. y Zacatelco, F. (2013). Creatividad gráfica y trabajo cooperativo en alumnos sobresalientes a través del teatro. En J. Gázquez, M. Pérez, M. Molero y R. Parra (Comps.). Investigación en el ámbito escolar: un acercamiento multidimensional a las variables psicológicas y educativas (137-140). GEU Editorial.

Tourón, J. (2020). Las altas capacidades en el sistema educativo español: reflexiones sobre el concepto y la identificación. Revista de Investigación Educativa, 38(1), 15-32. https://revistas.um.es/rie/article/view/396781/2 75221
Tuyub, J., Espíndola, M., Roque, J., González M., Cruz, M. (2016). Identificación y atención a niños con aptitudes sobresalientes en el estado de Hidalgo. En F. Herrera y M. Fajardo (eds.), la enseñanza y aprendizaje de las matemáticas en la formación inicial y la educación básica volumen I: memorias del ler congreso nacional de matemáticas, su enseñanza y aprendizaje "MATHENSM 2016" (240-243). https://www.researchgate.net/publication/31382 3239_Identificacion_y_atencion_a_ninos_con_ aptitudes_sobresalientes_en_el_estado_de_Hid algo

Valadez, D., Betancourt, J. Flores, J., Rodríguez, E. y Borges, A. (2020). Evaluation of the effects of grouping high capacity students in academic achievement and creativity. Sustainability, $\quad 12, \quad 1-20$. https://www.researchgate.net/publication/34182 7973_Evaluation_of_the_Effects_of_Grouping _High_Capacity_Students_in_Academic_Achie vement_and_Creativity

Valadez, D., Sánchez, P. Valdés, A. y Borges, A. (2015). Estratégias e programas para o desenvolvimiento da criatividade nas scolas do México [estrategias y programas para el desarrollo de la creatividad en las escuelas de México]. En M. Morais, L. Cerqueira de Miranda y S. Muglia (Ed.), Criatividade. Aplicacoes em contextos internacionais (257267). Vector Editora Psico-Pedagógica Ltda. https://www.researchgate.net/publication/29835 3651_Estrategias_e_programas_para_o_desenv olvimiento_da_criatividade_nas_scolas_do_Me xico

Valadez, M., Gutiérrez, S., Betancourt, J., Borges, A. y Galán, M. (2016). Atención educativa a alumnos y alumnas con aptitudes sobresalientes. Análisis de la propuesta de intervención de la secretaría de educación pública, México. Revista AMAzônica, 18(2), 237-259.

https://www.researchgate.net/publication/31985 2142_atencion_educativa_a_alumnos_y_alumn as_con_aptitudes_sobresalientes_analisis_de_la _propuesta_de_intervencion_de_la_secretaria_ de_educacion_publica_mexico_educational_att ention_to_students_with_outstan 
Verdín, A., Pintor, M., Gómez, M. (2012). Proceso de Aceleración en primaria. Caso de Nuevo León (tesis de maestría, Tecnológico de Monterrey).

https://www.researchgate.net/publication/27075 9493_Proceso_de_Aceleracion_en_Primaria_C aso_de_Nuevo_Leon

Villegas, I., Valadez, D., Borges, A., SolteroAvelar, R. y Zambrano, R. (2016). Evaluación del proceso de Acreditación, Promoción y Certificación Anticipada para niños con Aptitudes Sobresalientes Intelectuales. Revista Sobredotação, 131-144. https://www.aneis.org/wpcontent/uploads/2017/01/sobredotacao_2016.pd $\mathrm{f} \#$ page $=66$

Walker, C., Shore, B. y French, L. (2011). A theoretical context for examining students' preference across ability levels for learning alone or in groups. High Ability Studies, 22(1), 119-141. doi: http://dx.doi.org/10.1080/13598139.2011.5760 82

Zacatelco, F. Hernández, R. y Acle, G. (2013). Enriquecimiento de la creatividad escrita en alumnos sobresalientes de tercer ciclo de primaria. En G. Acle (coord.). Resiliencia en educación especial: una experiencia en la escuela regular (433-464). Gedisa.

Zacatelco, F. y Chávez, B. (2012). Evaluación de la eficacia de tres programas de enriquecimiento de la creatividad. Revista de Investigación y Divulgación en Psicología y Logopedia, 2(2), 35-40.

https://dialnet.unirioja.es/servlet/articulo?codig $\mathrm{o}=6369130$

Zacatelco, F., Chávez, B. y Lemus, A. (2017). Experiencia escrita a través del cuento. Programa para niños sobresalientes. Revista iberoamericana de psicología, 10(1), 9-18. https://www.researchgate.net/publication/33543 9923_Experiencia_escrita_a_traves_del_cuento _Programa_para_ninos_sobresalientes

Zambrano, R. (2017). El papel del profesorado de escuelas primarias en la propuesta nacional mexicana de atención a alumnos con aptitudes sobresalientes (tesis doctoral, Universidad de la Laguna).

https://www.educacion.gob.es/teseo/imprimirFi cheroTesis.do?idFichero=ykpG1DYEym4\%3D 